\title{
Patchouli oil ameliorates acute colitis: A targeted metabolite analysis of 2,4,6-trinitrobenzenesulfonic acid-induced rats
}

\author{
XIUTING YU ${ }^{1 *}$, GUANGHUA YANG ${ }^{2 *}$, HUA JIANG $^{3}$, SHUHAI LIN $^{4}$, YUHONG LIU $^{2}$, XIE ZHANG ${ }^{2}$, \\ HUIFANG ZENG ${ }^{1}$, ZIREN SU ${ }^{2}$, SONG HUANG ${ }^{2}$, LINLIN SHEN $^{3}$ and XIAOJUN ZHANG ${ }^{2}$ \\ ${ }^{1}$ Department of Pharmacy, The First Affiliated Hospital of Chinese Medicine, Guangzhou University of Chinese Medicine, \\ Guangzhou, Guangdong 510405; ${ }^{2}$ School of Chinese Materia Medica, Guangzhou University of Chinese Medicine, \\ Guangzhou, Guangdong 510006; ${ }^{3}$ Analysis Center of Shimadzu Enterprise Management (China) Co., Ltd., \\ Guangzhou, Guangdong 510010; ${ }^{4}$ School of Chinese Medicine, Hong Kong Baptist University, \\ Hong Kong 999077, Hong Kong SAR, P.R. China
}

Received September 16, 2015; Accepted October 28, 2016

DOI: $10.3892 /$ etm.2017.4577

\begin{abstract}
The incidence of inflammatory bowel disease (IBD), characterized by chronic, relapsing intestinal inflammation, has continually increased in recent years. A previous study by our group identified five potential metabolic markers possibly associated with the pathology of 2,4,6-trinitrobenzenesulfonic acid (TNBS)-induced IBD in rats. The present study aimed to examine the potential therapeutic effects of the essential oil of Pogostemon cablin (also known as patchouli; PO) on TNBS-induced rats and investigate the concomitant metabolic changes by targeting the previously identified potential markers. Pogostemon cablin is widely used to treat gastrointestinal diseases, including IBD, in China. The results of the present study showed that PO (270 mg/kg, rectal instillation) significantly alleviated colonic damage and reduced disease activity indicators and colonic myeloperoxidase in TNBS-induced rats. In addition, a targeted metabolic profiling study identified that four metabolites were elevated in the urine of the animals in the TNBS group, which were significantly inhibited by treatment with PO: Two tryptophan metabolites [4-(2-aminophenyl)-2,4-dioxobutanoic
\end{abstract}

Correspondence to: Dr Xiaojun Zhang, School of Chinese Materia Medica, Guangzhou University of Chinese Medicine, 232 Waihuandong Road, Guangzhou Higher Education Mega Center, Guangzhou, Guangdong 510006, P.R. China

E-mail: zhangxj@gzucm.edu.cn

Dr Linlin Shen, Analysis Center of Shimadzu Enterprise Management (China) Co., Ltd., 109 Liuhua Road, Guangzhou, Guangdong 510010, P.R. China

E-mail: skcsl1@shimazdu.com.cn

*Contributed equally

Key words: patchouli oil, 2,4,6-trinitrobenzenesulfonic acid, colitis, targeted metabolomics, tryptophan catabolites, gut microbial metabolites acid and 4,6-cihydroxyquinoline] and two gut microbial metabolites (phenylacetylglycine and p-cresol glucuronide). Taken together, these findings suggested that PO ameliorated the symptoms of TNBS-induced IBD and reversed the metabolic changes potentially associated with TNBS-induced IBD in rats.

\section{Introduction}

In recent years, there has been an increasing incidence of inflammatory bowel diseases (IBD), broadly classified as ulcerative colitis (UC) or Crohn's disease (CD) (1). Affected patients frequently experience diarrhea, abdominal pain and rectal bleeding, due to aberrant intestinal inflammation. These chronic and relapsing bowel-inflammatory symptoms greatly decrease patient quality of life (2). However, IBD remains largely incurable, since the individual's genetic susceptibility, external environment, intestinal microbial flora and immune responses are all involved and are functionally integrated in the etiology of IBD (3). Pharmaceutical treatments for IBD are classified into five major categories, namely anti-inflammatory drugs, immunosuppressants, biological agents, antibiotics and drugs for symptomatic relief (4). Unfortunately, the effects of these treatments are far from satisfying.

Pogostemonis Herba, the dried aerial part of Pogostemon cablin (Blanco) Benth. (Labiatae), commonly known as Cablin Patchouli, has been traditionally used as a principal component of a variety of renowned Chinese medicinal formulae, such as Baoji Pill and Huoxiangzhengqi capsule, to treat gastrointestinal diseases involving diarrhea, abdominal pain, vomiting and IBD. Patchouli oil (PO) is the essential oil produced by hydro-distilling the dry leaves of Pogostemon cablin. Studies have proven that PO has multiple activities, including anti-inflammatory (5), immunomodulatory (6) and antimicrobial actions (7). Thus, in the present study, it was speculated that PO may alleviate IBD by attenuating inflammation and inhibiting intestinal microorganisms.

Currently used animal models of IBD include transgenic, congenic, chemically induced and adoptive cell-transfer models (8), in which rodent colitis induced by 
2,4,6-trinitrobenzenesulfonic acid (TNBS) by stimulation of transmural oxidative stress and inflammatory factors is one of the most widely used models that closely resembles the pathogenesis of CD (9). In a previous metabolic profiling study by our group, five urinary metabolites potentially associated with the pathology of TNBS-induced colitis were identified using ultra-fast liquid chromatography-ion trap quadrupole time of flight mass spectrometry (UFLC-IT-QTOF-MS) (10).

The purpose of the present study was to investigate the potential therapeutic effect of $\mathrm{PO}$ on TNBS-induced rat colitis. The effect of PO was examined by evaluating the disease activity index (DAI), macroscopic and microscopic colonic damage scores and the myeloperoxidase (MPO) activity in the colon. Furthermore, a targeted metabolite analysis using the UFLC-IT-qTOF-MS approach was performed to characterize the metabolic changes in rat urine that accompanied the amelioration of the disease. The present study not only confirmed previously identified potential metabolic markers, but also suggested that the therapeutic effect of PO is closely associated with the adjustment of tryptophan metabolism and modulation of intestinal microorganisms.

\section{Materials and methods}

Animals. A total of 50 male Sprague-Dawley rats (age, 6-7 weeks; weight, $220 \pm 20 \mathrm{~g}$ ) were purchased from the animal center of Guangzhou University of Chinese Medicine (Guangzhou, China) and experimental protocols were approved by the Committee for Animal Care and Use at Guangzhou University of Chinese Medicine [project identification code, SYXK (yue) 2013-0085]. All animals received humane care in accordance with the Guide for the Care and Use of Laboratory Animals, published by the US National Institutes of Health (Bethesda, MD, USA). Animals were housed at a temperature of $23 \pm 2^{\circ} \mathrm{C}$ and humidity of $55 \pm 10 \%$ in a specific pathogen-free environment with a 12-h light/dark cycle and given free access to a standard laboratory diet and water. Prior to the start of the experiment, mice were acclimatized for at least one week.

Chemical reagents and materials. PO was purchased from Nanhai Zhongnan Co., Ltd. (Foshan, China; Lot 140801) and its quality was confirmed by gas chromatography-flame ionization detection in a previous study by our group (11). Chloral hydrate, TNBS, formic acid and D,L-4-chlorophenylalanine were purchased from Sigma-Aldrich (Merck Millipore, Darmstadt, Germany). $N$-phenylacetylglycine was purchased from Toronto Research Chemicals Inc. (Toronto, ON, Canada). All other chemicals were of analytical grade.

Drug administration and dose selection. Following 1 week acclimatization, rats were randomly divided into five groups ( $n=10$ per group). Animals in the control and TNBS groups received intra-rectal instillation of vehicle $[0.5 \%(\mathrm{w} / \mathrm{v})$ aqueous sodium carboxy methylcellulose (CMC-Na)] once daily for 7 days, and animals in the $\mathrm{PO}$ group received parallel intra-rectal instillation of PO (at the dose of 65, 135 and $270 \mathrm{mg} / \mathrm{kg}$ dissolved in $3 \mathrm{ml}$ vehicle). The results showed that the effect of $\mathrm{PO}$ at 65 and $135 \mathrm{mg} / \mathrm{kg}$ did not achieve statistical significance (Fig. 1A). Therefore, the dose of $270 \mathrm{mg} / \mathrm{kg}$ was selected for the subsequent histological and metabolomics evaluations.

TNBS-induced colitis and sample collection. All rats were anesthetized with chloral hydrate $(140 \mathrm{mg} / \mathrm{kg}$, by intraperitoneal injection) purchased from Damao Chemical Reagent Factory (Tianjin, China). In the TNBS and PO groups, colitis was induced by intra-rectal administration of TNBS dissolved in $50 \%$ ethanol at $25 \mathrm{mg} / 3 \mathrm{ml} / \mathrm{kg}$ body weight, at $8 \mathrm{~cm}$ from the anal verge after anesthetization (12). Control rats received an intra-rectal injection of saline but not TNBS. The animals then received vehicle with or without PO for 7 subsequent days. On the 5 th day after colitis induction, rats were placed into metabolic cages for acclimatization and two days later, urine samples were collected from each animal. All collected urine samples were immediately stored at $-80^{\circ} \mathrm{C}$ for UFLC-IT-QTOF-MS [Infusion Pump (LC-20ADXR*2), Degasser (DGU-20A 3 ), Autosampler (SIL-20AC), Column Heaters (CTO-20AC), Ion trap-time-of-flight mass spectrometer (LCMS-IT-TOF), Profiling workstation (LCMS-solution Ver. 3.6)] analysis (Shimadzu Corporation, Kyoto, Japan). Animals were sacrificed with chloral hydrate $(0.1 \mathrm{~g} / \mathrm{ml}$, by intraperitoneal injection, Damao Chemical Reagent Factory). After macroscopic scoring, the colonic segments were immediately removed following longitudinal opening and then rinsed with ice-cold physiological saline, blotted on filter paper and excised in two sections. One section was fixed in $10 \%$ buffered formalin at $4^{\circ} \mathrm{C}$ for $24 \mathrm{~h}$, embedded in paraffin, sectioned at $5-\mu \mathrm{m}$ thickness and finally stained with hematoxylin and eosin for routine histological examination performed with a microscope (BH22; Olympus Corporation, Tokyo, Japan). The other section was frozen in liquid nitrogen and stored at $-80^{\circ} \mathrm{C}$ for measurement of MPO activity.

Assessment of colonic damage. During the experimental period, animals were observed daily. Each day, the DAI scores were calculated by scoring body weight loss, trait of stool and hematochezia according to the classic scoring system by Cooper et al (13). For occult blood testing, a Hemoccult Sensa (REF 64151; Beckman Coulter, Brea, CA, USA) was used. In addition, a combination of micro- and macroscopic score, applied in order to estimate the degree of colitis, were determined by an observer who was blinded to the grouping according to previously established criteria (14). Each specimen was opened longitudinally and examined for immediate macroscopic scoring of injuries according the following criteria: 0 , normal appearance; 1 , focal hyperemia, no ulcers; 2 , ulceration with inflammation at 1 site; 3 , two or more sites of ulceration and inflammation; 4, major sites of damage extending $>1 \mathrm{~cm}$ along length of colon; 5-10, when an area of damage extended $>2 \mathrm{~cm}$ along the length of the colon, the score was increased by 1 for each additional $\mathrm{cm}$ of involvement. Colon sections were embedded in paraffin and cut into sections $5-\mu \mathrm{m}$ thick, then stained with hematoxylin and eosin for routine histological examination. Colonic mucosal damage in the histology specimens was assessed using previously established histological scoring criteria (14). The MPO activity was measured using a modified previous method (15) as follows: Pre-weighed colonic tissues $(0.1 \mathrm{~g})$ were cut into pieces and homogenized with an Ultra Turrax 
(T18 Basic; IKA, Staufen, Germany) in $0.5 \mathrm{ml}$ ice-cold $50 \mathrm{mM}$ phosphate-buffered saline (PBS; pH 6.0) containing 0.5\% hexadecyltrimethylammonium bromide (Sigma-Aldrich; Merck Millipore) at $4^{\circ} \mathrm{C}$. The homogenized samples were freeze-thawed and sonicated three times for $20 \mathrm{sec}$ each, followed by centrifugation at $3,000 \mathrm{x}$ g for $20 \mathrm{~min}$ at $4^{\circ} \mathrm{C}$. Subsequently, $20 \mu \mathrm{l}$ resulting supernatant was mixed with $180 \mu \mathrm{l} 50 \mathrm{mM}$ PBS containing $0.167 \mathrm{~g} / \mathrm{l} O$-dianisidine hydrochloride (Sigma-Aldrich; Merck Millipore) and $0.005 \mathrm{~g} / 1$ $\mathrm{H}_{2} \mathrm{O}_{2}$. The absorbance of the mixture was measured at $460 \mathrm{~nm}$ on a spectrophotometer (RT2100C; Rayto, Shenzhen, China) within $2 \mathrm{~min}$. MPO activity was expressed as units/mg wet tissue.

Urine sample preparation and UFLC-IT-qTOF-MS analysis. For UFLC/MS analysis, urine samples were prepared following a method described in a previous study by our group (10). In brief, each fresh urine sample was centrifuged at $1,400 \times g$ and the supernatant was collected and stored at $20^{\circ} \mathrm{C}$ until use. Urine samples $(200 \mu \mathrm{l})$ were mixed with $1.2 \mathrm{ml}$ organic solvent mixture [acetonitrile-methanol (3:1), spiked with $20 \mu \mathrm{g}$ of internal standard D,L-4-chlorophenylalanine] and centrifuged at $18,000 \mathrm{x} g$ at $4^{\circ} \mathrm{C}$ for $10 \mathrm{~min}$ for de-proteinization. The supernatant was dried under a flow of nitrogen to remove organic solvents and was then lyophilized. The residue was stored at $-4^{\circ} \mathrm{C}$ and reconstituted in $500 \mu \mathrm{l}$ methanol prior to analysis. Chromatographic separations of urine were performed on a Shim-pack XR-ODS III chromatographic column $(2.2 \mu \mathrm{m}, 2.0 \times 150 \mathrm{~mm}$; Shimadzu Corporation) using a Prominence UFLCXR system (Shimadzu Corporation). The column was maintained at $40^{\circ} \mathrm{C}$ and eluted with a linear gradient of $5-90 \% \mathrm{~B}$, where $\mathrm{A}=$ water with $0.1 \%$ formic acid and $\mathrm{B}=$ acetonitrile. The gradient program was as follows: 0-1 $\min , 5 \%$ B; $1-15 \min , 5-50 \%$ B; $15-18 \mathrm{~min}, 50-90 \%$ B; $18-20 \mathrm{~min}, 90 \% \mathrm{~B} ; 20.5-24 \mathrm{~min}, 5 \% \mathrm{~B}$; flow rate, $300 \mu \mathrm{l} / \mathrm{min}$. The injection volume was $5 \mu \mathrm{l}$. The column eluent was analyzed by QTOF mass spectrometry using positive and negative ion electro spray ionization (ESI). The column eluent was directed to the mass spectrometer without split. All MS and $\mathrm{MS}^{\mathrm{n}}$ mass spectra were acquired on an LCMS-IT-TOF mass spectrometer (Shimadzu Corporation) operating in either positive or negative ESI mode. The desolvation gas was set to $10 \mathrm{l} / \mathrm{min}$ at a temperature of $200^{\circ} \mathrm{C}$. The cone gas was set to $1.5 \mathrm{l} / \mathrm{min}$ and the source temperature was set to $200^{\circ} \mathrm{C}$. The voltage of the ESI source and detector were $+4.5,-3.5$ and $1.60 \mathrm{kV}$, respectively. All data were collected in the full scan mode $(100-1,000 \mathrm{~m} / \mathrm{z})$. The dwell time for each scan was set to $50 \mathrm{msec}$.

Data processing and multivariate data analysis. The LC-IT-QTOF-MS raw data were pre-processed using Profiling solution software ver. 3.6 (Shimadzu Corporation). The resulting multi-dimensional data, comprising peak number [retention time (RT)-m/z pair], sample name (observation) and ion intensity (variable) were acquired as multivariate data matrices and exported into SIMCA-P ver. 10.0 (Umetrics $\mathrm{AB}$, Umea, Sweden) for multivariate data analysis. The ion intensities for each peak detected were then normalized, within each sample, to the sum of the peak intensities in that sample. The relative intensities of targeted metabolites were then normalized to that of the spiked internal standard D,L-4-chlorophenylalanine. Pareto-scaling and supervised partial least squares-discriminate analyses (PLS-DA) were selected for data mining and pattern recognition after optimization.

Statistical analysis. Values are expressed as the mean \pm standard deviation and analyzed with SPSS software version 17.0 (SPSS, Inc., Chicago, IL, USA). The groups were compared by one-way analysis of variance followed by the Dunnett's test and statements of statistical significance were based on $\mathrm{P}<0.05$.

\section{Results}

Pathological characteristics of PO-treated rats. As shown in Fig. 1A, the TNBS group had a significantly higher $(\mathrm{P}<0.05)$ colonic damage score and DAI for CD (16) than the control group. These markedly decreased following PO treatment, particularly at the dose of $270 \mathrm{mg} / \mathrm{kg}$. In addition, the representative images show that rats in the control group displayed healthy colons without ulceration, while obvious tissue necrosis was found in the colon of TNBS-treated rats (Fig. 1B). Of note, PO attenuated the symptoms of ulceration. Moreover, as indicated in Fig. 2A, the PO group showed significantly lower histological scores and MPO activity, an index of neutrophil accumulation in the tissue with correlates with severity (17), when compared with the TNBS-treated group. Furthermore, the control group maintained an intact architecture of the colon (Fig. 2B), whereas TNBS-treated rats revealed significant necrosis of the mucosa and submucosa and dense inflammatory-cell infiltration in the submucosa and muscularis mucosae. However, PO treatment attenuated inflammatory-cell infiltration. These results confirmed that TNBS induced serious colitis in rats, while rectal application of PO significantly attenuated the symptoms of colitis.

Targeted metabolite analysis of urine from rats by LC-IT-QTOF-MS. The differences in base peak intensity chromatographs of urine samples from the control, TNBS and PO-treated groups were displayed in ESI positive (Fig. 3A-C) and negative mode (Fig. 4A-C). In order to clearly differentiate between groups, PLS-DA was used to investigate the fluctuation of metabolites. As shown in Fig. 5A-D, urine samples from the TNBS group were distinctly separated from the control and PO groups in the ESI positive as well as negative mode.

Identification of the targeted metabolites in urine. A previous study by our group has identified five endogenous metabolites that are potential metabolic markers of TNBS-induced IBD in rats (10). Therefore, in the present study, the data of the targeted metabolites we extracted from the urine data matrix and the variable importance in the projection (VIP) was calculated following PLS-DA processing. Then Student's $t$-test was used to select potential metabolites worthy of preferential study. $\mathrm{P}<0.05$ was considered to indicate statistical significance which was assessed using SPSS 17.0. Identification of the five endogenous metabolites was performed based on the retention time, accurate mass by MS and MS/MS, as well as 

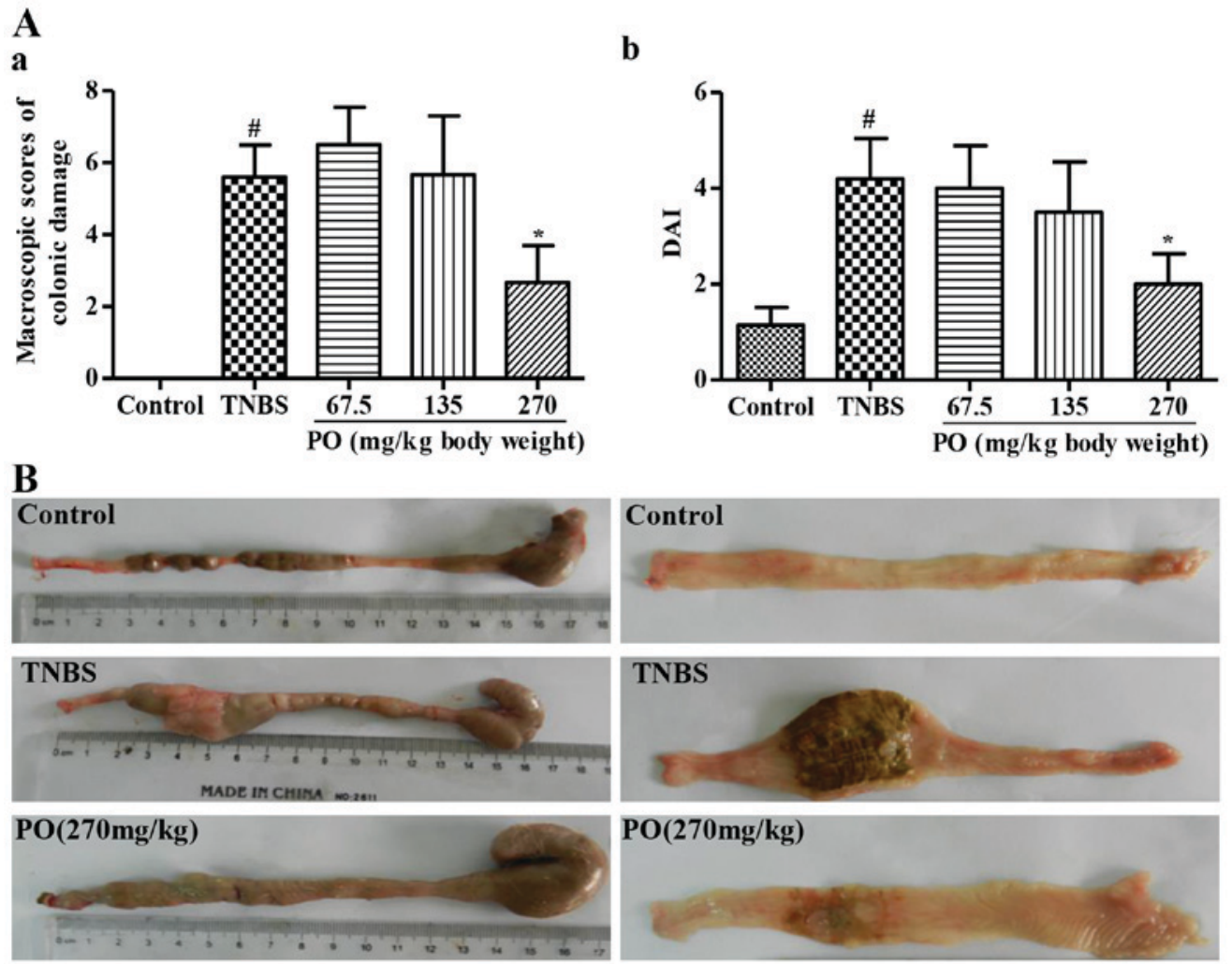

Figure 1. Macroscopic characteristics of rats in the Control, TNBS and PO group. (A-a) Macroscopic scores of colonic damage. (A-b) DAI. Values are expressed as the mean \pm standard deviation $(\mathrm{n}=7-10)$. ${ }^{~} \mathrm{P}<0.05$ vs. Control group; $\mathrm{P}<0.05$ vs. TNBS group. (B) Images of macroscopic colon damages prior to (left) and after (right) longitudinal opening. DAI, disease activity index; TNBS, 2,4,6-trinitrobenzenesulfonic acid; PO, patchouli oil.

A a

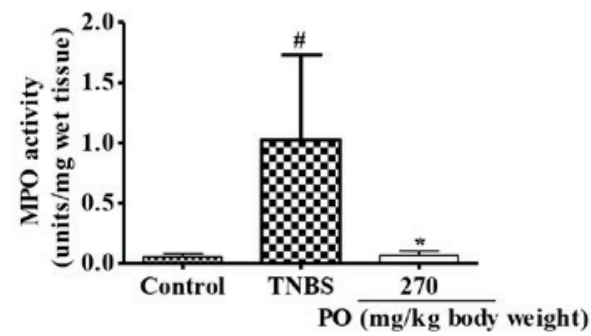

b

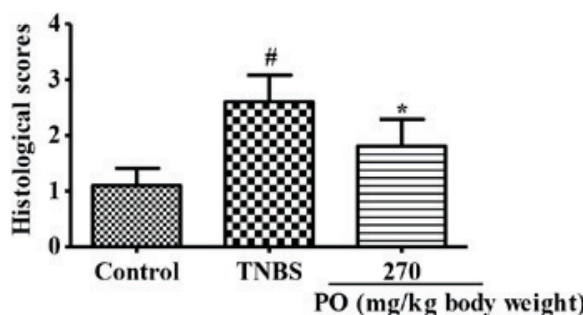

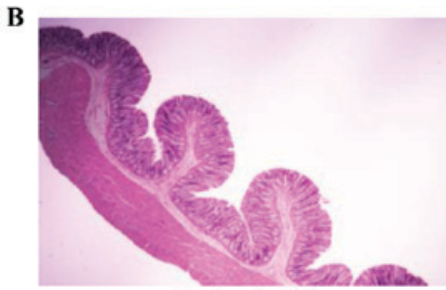

Control $x 40$

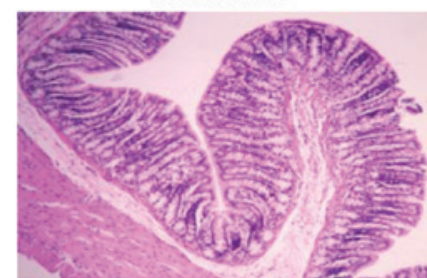

Control x100

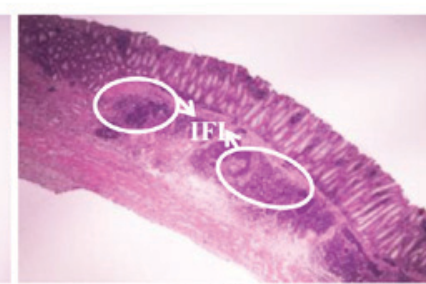

TNBS $\times 40$

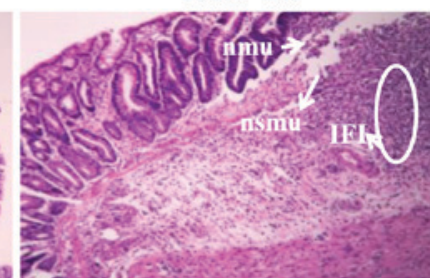

TNBS $\mathbf{x 1 0 0}$

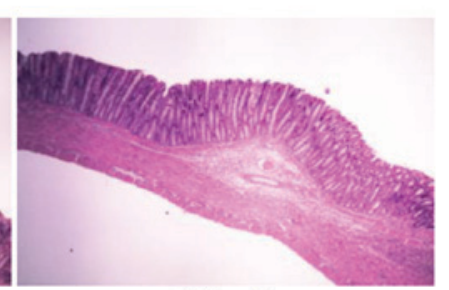

PO $\mathrm{x} 40$

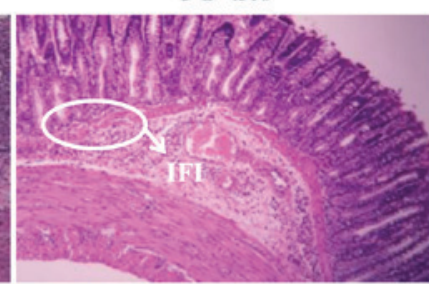

PO x100

Figure 2. Microscopic characteristics of rats in Control, TNBS and PO groups. (A-a) MPO activity. (A-b) Histological scores. Values are expressed as the mean \pm standard deviation $(\mathrm{n}=7-10)$. ${ }^{*} \mathrm{P}<0.05$ vs. Control group; ${ }^{*} \mathrm{P}<0.05$ vs. TNBS group. (B) Images showing histological changes. The control group showed normal features of mucosa with intact epithelial surface, submucosa and muscularis layer. The TNBS group showed mucosal erosion with loss of epithelial cells, focal nmu and nsmu and dense IFI. The PO group had nearly intact mucosa and decreased inflammatory cells. TNBS, 2,4,6-trinitrobenzenesulfonic acid; PO, patchouli oil; nmu, necrosis of mucosa; nsmu, necrosis of submucosa; IFI, inflammatory-cell infiltration. 

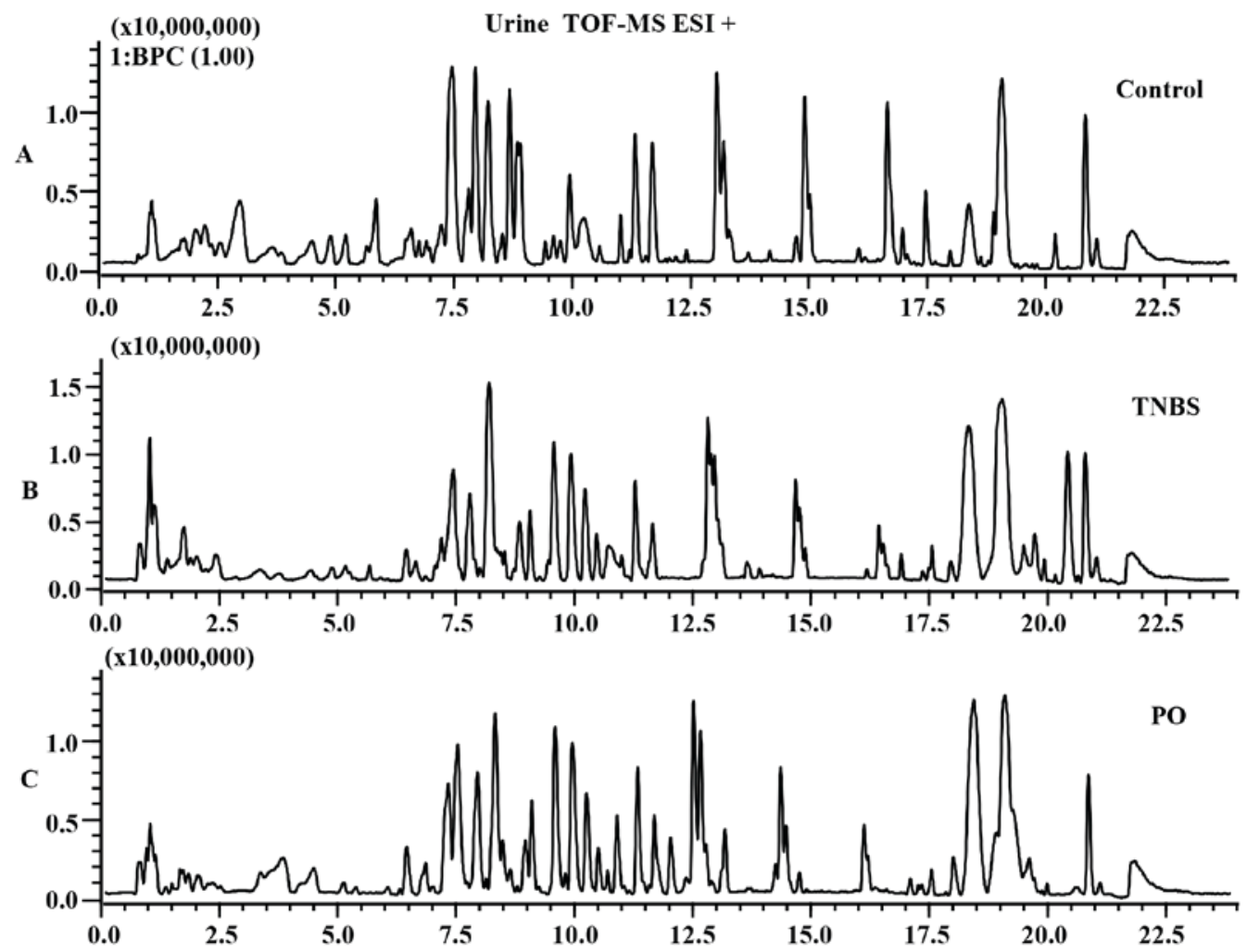

Figure 3. BPC of urinary samples generated using ultra-fast liquid chromatography-ion trap quadrupole TOF-MS. BPCs of urine samples from rats in (A) the Control, (B) TNBS and (C) PO group recorded in positive mode. BPC, base peak intensity chromatograms; TOF-MS ESI, time of flight mass spectrometry electron spray ionization.
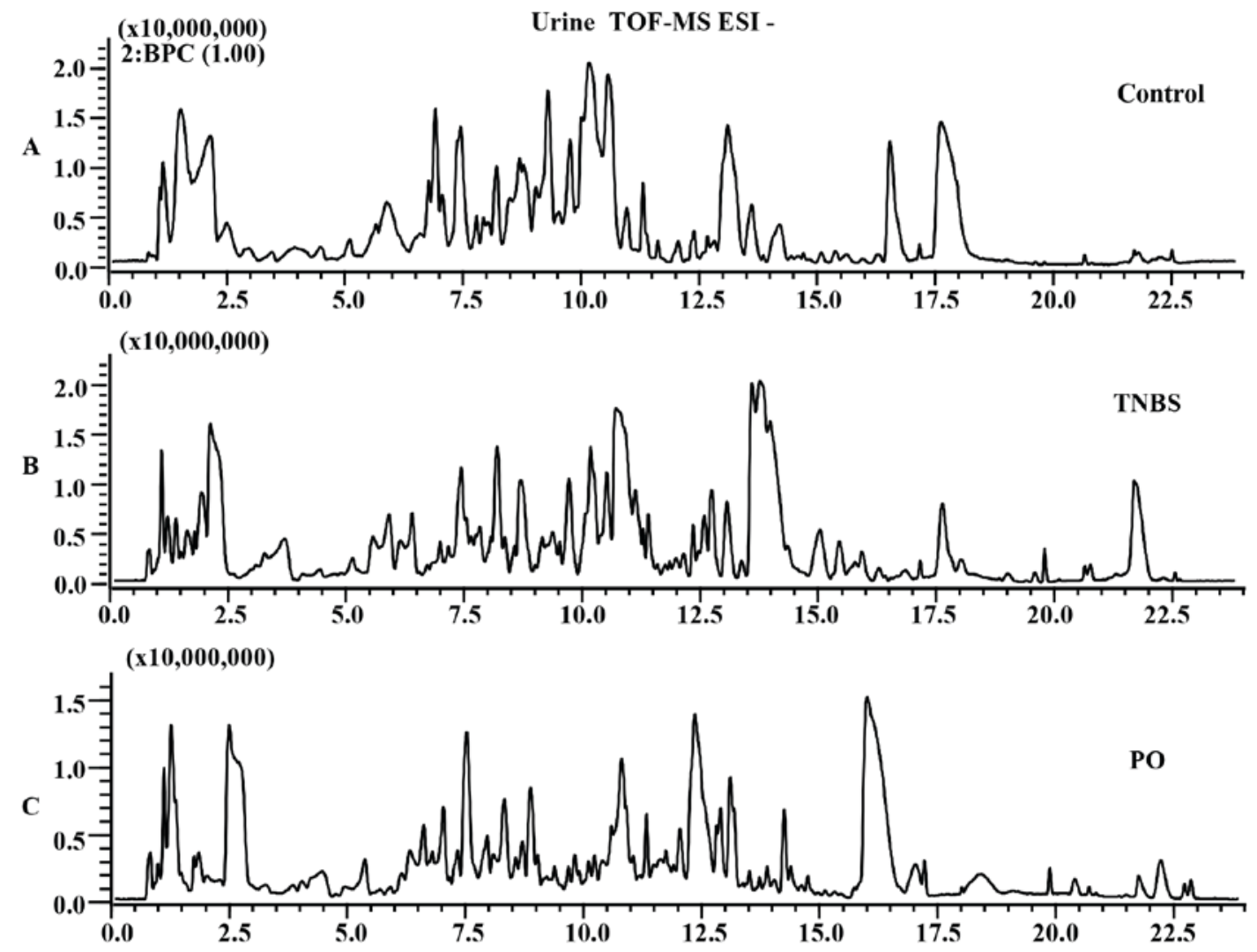

Figure 4. BPC of urinary samples generated using ultra-fast liquid chromatography-ion trap quadrupole TOF-MS. BPCs of urine samples from rats in (A) the Control, (B) TNBS and (C) PO group recorded in negative mode. BPC, base peak intensity chromatograms; TOF-MS ESI, time of flight mass spectrometry electron spray ionization. 

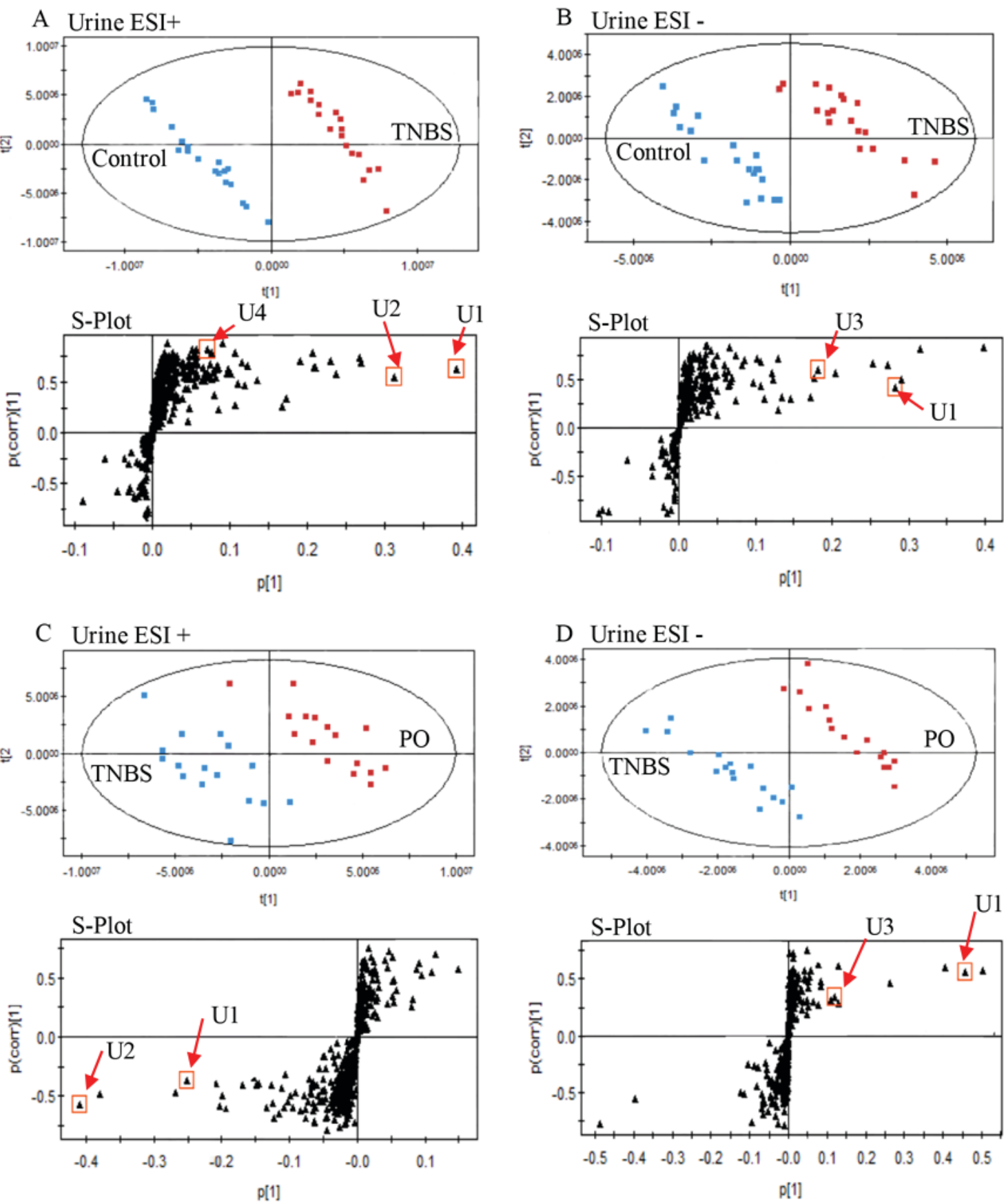

Figure 5. Targeted metabolic analysis of urinary samples using ultra-fast liquid chromatography-ion trap quadrupole time of flight mass spectrometry-based metabolomics. PLS-DA score plots and PLS S-plot based on detected urinary metabolites from TNBS-induced colitis and control rats in (A) positive and (B) negative ion mode. PLS-DA score plots and PLS S-plot based on detected urinary metabolites from TNBS-induced colitis and PO-treated rats in (C) positive and (D) negative ion mode. The black circles represent the retention time and $\mathrm{m} / \mathrm{z}$ data pairs for each metabolite. Metabolites: U1, phenylacetylglycine; U2, 4,6-cihydroxyquinoline; U3, p-cresol glucuronide; U4, 4-(2-aminophenyl)-2,4-dioxobutanoic acid. ESI, electron spray ionization; PO, patchouli oil; PLS-DA, partial least squares-discriminate analysis; TNBS, 2,4,6-trinitrobenzenesulfonic acid.

elemental composition data by comparing results from various databases such as the Human Metabolome Database (HMDB; http://www.hmdb.ca), the METLIN Metabolite Database (http://metlin.scripps.edu) and Mass Bank (http://www. massbank.jp) (10). The results of the analysis are shown in Table I. A total of four metabolites [U1, phenylacetylglycine; U2, 4,6-cihydroxyquinoline; U3, p-cresol glucuronide; U4, 4-(2-aminophenyl)-2,4-dioxobutanoic acid] were identified in urine samples from the different groups in positive ESI and/or negative ESI modes (VIP value $>1$ ). Metabolites U1 and U3 are associated with the homeostasis of intestinal microbiota, while U2 and U4 are tryptophan metabolites (10). As shown in Fig. 6, the levels of U1-U4 were significantly higher in the TNBS-treated rats than in the control rats. By contrast, PO-treatment significantly reversed the increases in the levels of metabolites U1-U4 that occurred in TNBS mice. Levels of metabolite U4 in the PO-treated group was significantly decreased to levels below the limit of detection. 


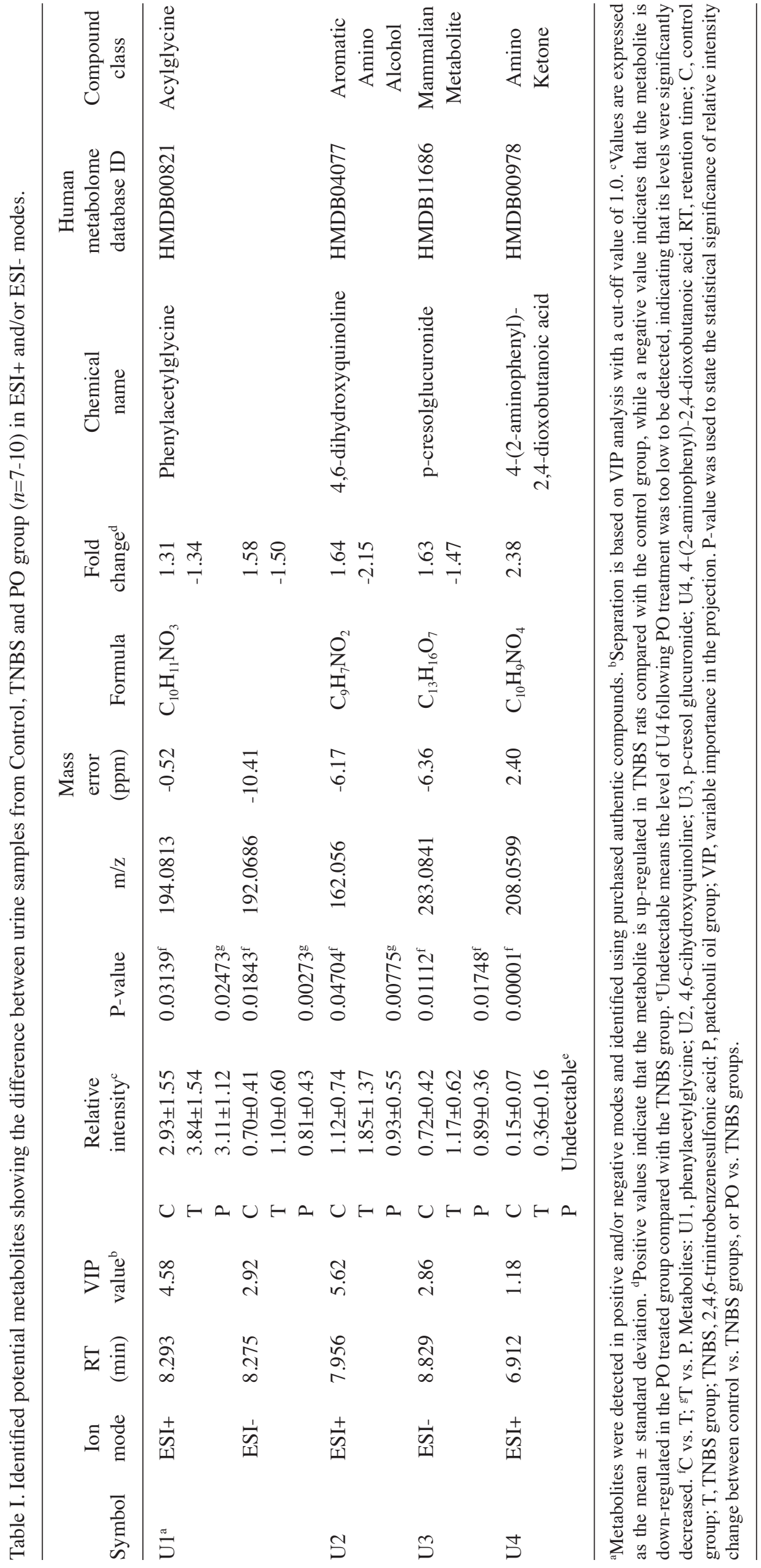




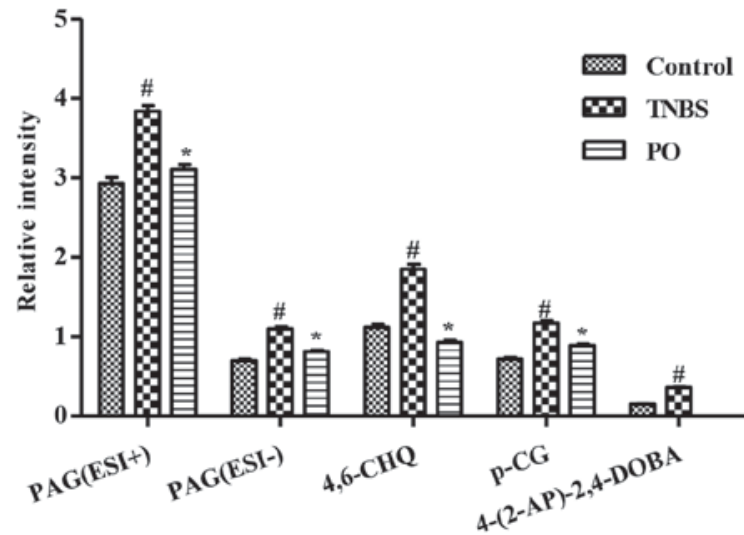

Figure 6. Relative intensity of targeted metabolites in urinary samples of Control, TNBS and PO groups. Intensity of metabolites in ESI-positive and/or ESI-negative modes used in this figure were presented as the mean \pm standard error of the mean $(\mathrm{n}=7-10) .{ }^{\text {" }} \mathrm{P}<0.05$ vs. Control group; ${ }^{*} \mathrm{P}<0.05$ vs TNBS group. PAG (U1), phenylacetylglycine; 4,6-CHQ (U2), 4,6-cihydroxyquinoline; p-CG (U3), p-cresolglucuronide; 4-(2-AP)-2,4-DOBA (U4), 4-(2-aminophenyl)-2,4-dioxobutanoic acid; ESI, electron spray ionization; PO, patchouli oil; TNBS, 2,4,6-trinitrobenzenesulfonic acid.

\section{Discussion}

The etiology of IBD involves a complex interaction between the genetic, environmental or microbial factors and the immune responses, which eventually result in recurrent diarrhea, rectal inflammatory lesions and bleeding (18). PO, the essential oil of Pogostemon cablin, possesses anti-inflammatory, immunomodulatory and anti-microbial activities, indicating a potential for the symptomatic ease of IBD. The present study demonstrated for the first time that rectal administration of PO downregulates the macroscopic and histological colonic damage scores, DAI and colonic MPO activities of rats with TNBS-induced colitis. Furthermore, a targeted metabolic analysis of urine samples based on a high-throughput UFLC-IT-QTOF-MS approach showed that PO decreased the levels of four differential metabolites (two gut microbial metabolites and two tryptophan metabolites), which were markedly increased following treatment with TNBS.

As mentioned previously, one of the major pathological factors of IBD is the commensal bacterial flora (19). The microbial dysbiosis may interplay with the damage of the intestinal barrier and the disturbance of the mucosal immune system, inducing destructive inflammatory immune responses, considered to be the basis of IBD pathogenesis (20). The present study demonstrated that the levels of two gut microbial metabolites, phenylacetylglycine and p-cresol glucuronide, were upregulated in the urine of TNBS rats. Phenylacetylglycine is a gut microbial co-metabolite (21), generated by conjugation of glycine with phenylacetate, via the phase-II detoxification mechanism in the liver or the gut mucosa (22). P-cresol glucuronide is a glucuronide derivative of p-cresol, a metabolic product of Clostridium difficile in the large intestine (23). Consistent with previous studies, the observed increases in the output of those 2 metabolites indicated dysbiosis of the intestinal microbiota (24). However, this change was significantly attenuated by PO. As PO has marked anti-bacterial activity (7), it may be deduced that PO possibly ameliorated TNBS-induced colitis via regulating intestinal microorganisms.

In addition,IBD is initiated and perpetuated by adysregulated immune response to antigens (25). The present study identified two tryptophan metabolites that are closely involved in immune regulation and the inflammatory response. Tryptophan has a pivotal role in immunosuppression in inflammatory diseases, immune system regulation, protein synthesis as well as serotonin (5-HT) and melatonin production $(26,27)$. The present study further identified 2 metabolites associated with the homeostasis of intestinal microbiota [4-(2-aminophenyl)-2,4-dioxobutanoic acid and 4,6-cihydroxyquinoline] in urine. The former is the intermediate product in metabolism of kynurenine, which regulates the immune response (28). The latter is an intermediate metabolite of 5-hydroxytryptophan, a precursor of 5-HT, which activates immune cells to produce pro-inflammatory mediators (29). Hence, the increased urine levels of these two metabolites suggest accelerated tryptophan-kynurenine and tryptophan-5-HT metabolism. The enhanced tryptophan and 5-HT metabolism may be associated with immune dysfunction in IBD (30).

In conclusion, the results of the present study demonstrated that treatment with $270 \mathrm{mg} / \mathrm{kg}$ via rectal instillation significantly ameliorated TNBS-induced rat colitis and significantly reversed the metabolic changes of four metabolites in the urine of the TNBS rats, which were two tryptophan metabolites [4-(2-aminophenyl)-2,4-dioxobutanoic acid and 4,6-cihydroxyquinoline] and two gut microbial metabolites (phenylacetylglycine and p-cresol glucuronide). These metabolic changes may be associated with TNBS-induced colitis in rats.

\section{Acknowledgements}

The present study was supported by grants from the National Natural Science Foundation of China (project nos. 81303200, 21377106 and 81173534), Guangdong Natural Science Foundation (project nos. S2013010016627 and S2012010008893), Medical Scientific Research Foundation of Guangdong Province (project no. A2013232), Administration of Traditional Chinese Medicine of Guangdong Province (project no. 20132142), the Special Funds from Central Finance of China in Support of the Development of Local Colleges and University (project no. 276, 2014), the China state '12th Five year Plan' Scientific and Technological Support Scheme (project no. 2012BAI029B09), Hong Kong, Macao and Taiwan Science \& Technology Cooperation Program of China (no. 2014DFH30010) and the Guangdong Province Universities and Colleges Pearl River Scholar Funded Scheme (2011). The authors gratefully acknowledge this financial support.

\section{References}

1. Frolkis A, Dieleman LA, Barkema HW, Panaccione R, Ghosh S, Fedorak RN, Madsen K and Kaplan GG; Alberta IBD Consortium: Environment and the inflammatory bowel diseases. Can J Gastroenterol 27: e18-e24, 2013.

2. Maloy KJ and Powrie F: Intestinal homeostasis and its breakdown in inflammatory bowel disease. Nature 474: 298-306, 2011. 
3. Danese S and Fiocchi C: Etiopathogenesis of inflammatory bowel diseases. World J Gastroenterol 12: 4807-4812, 2006.

4. Triantafillidis JK, Merikas E and Georgopoulos F: Current and emerging drugs for the treatment of inflammatory bowel disease. Drug Des Devel Ther 5: 185-210, 2011.

5. Xian YF, Suo J, Huang XD, Hou SZ, Chen, J Ye M and Su ZR: A Pharmacological Study on Anti-inflammatory Effects of Refined Huodan Recipe. Zhong Guo Shi Yan Fang Ji Xue Za Zhi She 13: 54-56, 2007 (In Chinese)

6. Qi SS, Hu LP, Chen WN, Sun HB and Ma XD: Immunological regulation effects of essential oil in leaves of Cablin Patchouli herbal on mice. Chin Arch Trad Chin Med 27: 774-776, 2009.

7. Yang X, Zhang X, Yang SP and Liu WQ: Evaluation of the antibacterial activity of patchouli oil. Iran J Pharm Res 12: 307-316, 2013

8. Mizoguchi A and Mizoguchi E: Animal models of IBD: Linkage to human disease. Curr Opin Pharmacol 10: 578-587, 2010.

9. Lu X, Zhao X, Bai C, Zhao C, Lu G and Xu G: LC-MS-based metabonomics analysis. J Chromatogr B Analyt Technol Biomed Life Sci 866: 64-76, 2008.

10. Zhang XJ, Choi FF, Zhou Y, Leung FP, Tan S, Lin S, Xu H, Jia W, Sung JJ, Cai Z and Bian Z: Metabolite profiling of plasma and urine from rats with TNBS-induced acute colitis using UPLC-ESI-QTOF-MS-based metabonomics-a pilot study. FEBS J 279: 2322-2338, 2012.

11. Lin RF, Feng XX, Li CW, Zhang XJ, Yu XT, Zhou JY, Zhang X, Xie YL, Su ZR and Zhan JY: Prevention of UV radiation-induced cutaneous photoaging in mice by topical administration of patchouli oil. J Ethnopharmacol 154: 408-418, 2014.

12. McCafferty DM, Sharkey KA and Wallace JL: Beneficial effects of local or systemic lidocaine in experimental colitis. Am J Physiol 266: G560-G567, 1994.

13. Cooper HS, Murthy SN, Shah RS and Sedergran DJ: Clinicopathologic study of dextran sulfate sodium experimental murine colitis. Lab Invest 69: 238-249, 1993.

14. Gue M, Bonbonne C, Fioramonti J, Moré J, Del Rio-Lachèze C, Coméra C and Buéno L: Stress-induced enhancement of colitis in rats: CRF and arginine vasopressin are not involved. Am J Physiol 272: G84-G91, 1997.

15. Schicho R and Storr M: Topical and systemic cannabidiol improves trinitrobenzene sulfonic acid colitis in mice. Pharmacology 89: 149-155, 2012.

16. Best WR, Becktel JM, Singleton JW and Kern F Jr: Development of a Crohn's disease activity index. National cooperative crohn's disease study. Gastroenterology 70: 439-444, 1976.

17. Krawisz JE, Sharon P and Stenson WF: Quantitative assay for acute intestinal inflammation based on myeloperoxidase activity. Assessment of inflammation in rat and hamster models. Gastroenterology 87: 1344-1350, 1984.

18. Zhang YZ and Li YY: Inflammatory bowel disease: Pathogenesis. World J Gastroenterol 20: 91-99, 2014.
19. Guarner F: The intestinal flora in inflammatory bowel disease: Normal or abnormal? Curr Opin Gastroenterol 21: 414-418, 2005

20. Stephani J, Radulovic K and Niess JH: Gut microbiota, probiotics and inflammatory bowel disease. Arch Immunol Ther Exp (Warsz) 59: 161-177, 2011

21. Yap IK, Li JV, Saric J, Martin FP, Davies H, Wang Y, Wilson ID, Nicholson JK, Utzinger J, Marchesi JR and Holmes E: Metabonomic and microbiological analysis of the dynamic effect of vancomycin-induced gut microbiota modification in the mouse. J Proteome Res 7: 3718-3728, 2008

22. Akira K, Hichiya H, Morita M, Shimizu A and Mitome H: Metabonomic study on the biochemical response of spontaneously hypertensive rats to chronic taurine supplementation using (1)H NMR spectroscopic urinalysis. J Pharm Biomed Anal 85: 155-161, 2013.

23. Walsh MC, Brennan L, Pujos-Guillot E, Sébédio JL, Scalbert A, Fagan A, Higgins DG and Gibney MJ: Influence of acute phytochemical intake on human urinary metabolomic profiles. Am J Clin Nutr 86: 1687-1693, 2007.

24. Akira K, Masu S, Imachi M, Mitome H and Hashimoto T: A metabonomic study of biochemical changes characteristic of genetically hypertensive rats based on (1)H NMR spectroscopic urinalysis. Hypertens Res 35: 404-412, 2012.

25. Del Angel-Meza AR, Dávalos-Marín AJ, Ontiveros-Martinez LL, Ortiz GG, Beas-Zarate C, Chaparro-Huerta V, Torres-Mendoza BM and Bitzer-Quintero OK: Protective effects of tryptophan on neuro-inflammation in rats after administering lipopolysaccharide. Biomed Pharmacother 65: 215-219, 2011.

26. Kim CJ, Kovacs-Nolan JA, Yang C, Archbold T, Fan MZ and Mine Y: 1-Tryptophan exhibits therapeutic function in a porcine model of dextran sodium sulfate (DSS)-induced colitis. J Nutr Biochem 21: 468-475, 2010.

27. Frumento G, Rotondo R, Tonetti M, Damonte G, Benatti U and Ferrara GB: Tryptophan-derived catabolites are responsible for inhibition of $\mathrm{T}$ and natural killer cell proliferation induced by indoleamine 2,3-dioxygenase. J Exp Med 196: 459-468, 2002.

28. Nguyen NT, Kimura A, Nakahama T, Chinen I, Masuda K, Nohara K, Fujii-Kuriyama Y and Kishimoto T: Aryl hydrocarbon receptor negatively regulates dendritic cell immunogenicity via a kynurenine-dependent mechanism. Proc Natl Acad Sci USA 107: 19961-19966, 2010.

29. Linden DR, Chen JX, Gershon MD, Sharkey KA and Mawe GM: Serotonin availability is increased in mucosa of guinea pigs with TNBS-induced colitis. Am J Physiol Gastrointest Liver Physiol 285: G207-G216, 2003.

30. Gersemann M, Wehkamp J and Stange EF: Innate immune dysfunction in inflammatory bowel disease. J Intern Med 271: 421-428, 2012. 